\title{
Stability analysis of a diagonally implicit scheme of block backward differentiation formula for stiff pharmacokinetics models
}

\author{
Hazizah Mohd ljam', Zarina Bibi Ibrahim ${ }^{1,2^{*}}$, Zanariah Abdul Majid ${ }^{1,2}$ and Norazak Senu $u^{1,2}$
}

\section{"Correspondence:}

zarinabb@upm.edu.my

${ }^{1}$ Institute for Mathematical Research (INSPEM), Universiti Putra Malaysia (UPM), 43400 Selangor, Malaysia ${ }^{2}$ Department of Mathematics, Faculty of Science, Universiti Putra Malaysia (UPM), 43400 Selangor, Malaysia

\begin{abstract}
In this paper, we analyze the criteria for the stability of a method suited to the ordinary differential equations models. The relevant proof that the method satisfies the condition of stiff stability is also provided. The aim of this paper is therefore to construct an efficient two-point block method based on backward differentiation formula which is A-stable and converged. The new diagonally implicit scheme is formulated to approximate the solution of the pharmacokinetics models. By implementing the algorithm, the numerical solution to the models is compared with a few existing methods and established stiff solvers. It yields significant advantages when the diagonally implicit method with a lower triangular matrix and identical diagonal elements is considered. The formula is designed in such a way that it permits a maximum of one LU decomposition for each integration stage.
\end{abstract}

Keywords: Stiff ODEs; Block backward differentiation formula; Diagonally implicit; Stability; Pharmacokinetics models

\section{Introduction}

Initial value problems (IVPs) governed by the system of ordinary differential equations (ODEs) often arise in the modeling of physical, chemical, and biological systems. It has emerged widely in the areas of medical sciences such as epidemiology, cell physiology, and pharmacology. According to Gear in [1], due to the existence of greatly differing time constants in these systems, a phenomenon known as stiffness is exhibited. Such systems of equations are typically very stable, but often the conventional numerical methods are inefficient due to the severe step length restriction imposed by the numerical stability requirements (see [2]).

In this article, we consider the model of stiff ODEs system in the form of

$$
\widetilde{y}^{\prime}=A \widetilde{y}+\widetilde{\phi}(x), \quad \widetilde{y}(a)=\widetilde{\beta}, \quad a \leq x \leq b,
$$

where

$$
\widetilde{y}^{T}=\left(y_{1}, y_{2}, \ldots, y_{s}\right), \quad \widetilde{\beta}^{T}=\left(\beta_{1}, \beta_{2}, \ldots, \beta_{s}\right) \quad \text { and } \quad A \text { is an } s \times s \text { matrix. }
$$

(c) The Author(s) 2020. This article is licensed under a Creative Commons Attribution 4.0 International License, which permits use, sharing, adaptation, distribution and reproduction in any medium or format, as long as you give appropriate credit to the original author(s) and the source, provide a link to the Creative Commons licence, and indicate if changes were made. The images or other third party material in this article are included in the article's Creative Commons licence, unless indicated otherwise in a credit line to the material. If material is not included in the article's Creative Commons licence and your intended use is not permitted by statutory regulation or exceeds the permitted use, you will need to obtain permission directly from the copyright holder. To view a copy of this licence, visit http://creativecommons.org/licenses/by/4.0/. 
The eigenvalues $\lambda_{i}$ of the Jacobian matrix $J=\frac{\partial f}{\partial \widetilde{y}}$ of the system in (1), evaluated at $(x, \widetilde{y})$, will be used in the most widely heuristic definition of stiffness by Lambert in [3] as follows: The system in (1) is said to be stiff if

(i) $\operatorname{Re}\left(\lambda_{i}\right)<0, i=1,2, \ldots, s$, and

(ii) $\max _{i}\left|\operatorname{Re}\left(\lambda_{i}\right)\right| \gg \min _{i}\left|\operatorname{Re}\left(\lambda_{i}\right)\right|$ where the ratio $\left[\max _{i}\left|\operatorname{Re}\left(\lambda_{i}\right)\right|\right]$ : $\left[\min _{i}\left|\operatorname{Re}\left(\lambda_{i}\right)\right|\right]$ is called the stiffness ratio.

As stated in [4], a stiff ODEs system is characterized by the property that the ratio of the largest to the smallest eigenvalue is greater than one.

The general solution of (1) takes the form

$$
\widetilde{y}(x)=\sum_{i=0}^{s} \kappa_{i} e^{\lambda_{i} x} \widetilde{c}_{i}+\widetilde{\psi}(x)
$$

where $\kappa_{i}$ are the arbitrary constants, $\widetilde{c}_{i}$ are the eigenvectors of corresponding eigenvalues $\lambda_{i}$, and $\widetilde{\psi}(x)$ is a particular integral. Interpreting $x$ to be time, we denote the first term $\sum_{i=0}^{s} \kappa_{i} e^{\lambda_{i} x} \widetilde{c}_{i}$ as the transient solution and the remaining $\widetilde{\psi}(x)$ as the steady-state solution. Now, let us assume condition (i) is satisfied, which implies that the term $\sum_{i=0}^{s} \kappa_{i} e^{\lambda_{i} x} \widetilde{c}_{i} \rightarrow 0$ as $x \rightarrow \infty$. Let $\left|\operatorname{Re}\left(\lambda_{\mu}\right)\right|$ and $\left|\operatorname{Re}\left(\lambda_{v}\right)\right|$ be defined by

$$
\left|\operatorname{Re}\left(\lambda_{\mu}\right)\right| \geq\left|\operatorname{Re}\left(\lambda_{i}\right)\right| \geq\left|\operatorname{Re}\left(\lambda_{v}\right)\right|, \quad i=1,2, \ldots, s,
$$

so that $\kappa_{i} e^{\lambda \mu x} \widetilde{c}_{i}$ and $\kappa_{i} e^{\lambda_{v} x} \widetilde{c}_{i}$ are the fastest and slowest transient, respectively. If the system in (1) is solved numerically and aimed at achieving a steady-state phase, continuing integration is needed until the slowest transient is negligible. Therefore, the smaller $\left|\operatorname{Re}\left(\lambda_{v}\right)\right|$ is, the longer the integration period will be. However, for the larger $\left|\operatorname{Re}\left(\lambda_{\mu}\right)\right|$, a sufficiently small step length is required so that $h$ will lie within the region of absolute stability of the method (refer to $[2,3,5]$ ).

According to [6], due to their relative ease of execution, the subclass of diagonally implicit Runge-Kutta (DIRK) methods has become the most commonly used in solving stiff first order ODEs. The fully implicit Runge-Kutta (FIRK) and DIRK methods on Butcher array have the forms as tabulated in Table 1.

The coefficients of $a_{i j}$ in Table 1 constitute a matrix denoted as $A$. The method with a full coefficient matrix, i.e., FIRK requires to solve a system of ( $n$-dimensional $\times r$-stages) nonlinear equations in each of its integration stages [8]. To reduce the computational cost of evaluating the stages in the FIRK method, [7-11] opted for the DIRK method. As stated in [6], this method is characterized by a lower triangular $A$-matrix with $a_{i j}=0$ for $i<$ $j$ and is sometimes referred to as semi-implicit or semi-explicit Runge-Kutta methods. According to [8], Newton-type iteration is required when the problem is stiff in order to solve the linear systems at each stage with the coefficient matrix written in the form of

\begin{tabular}{|c|c|}
\hline (a) FIRK & (b) DIRK \\
\hline \begin{tabular}{l|lll}
$c_{1}$ & $a_{11}$ & $a_{12}$ & $a_{13}$
\end{tabular} & \begin{tabular}{l|lll}
$c_{1}$ & $a_{11}$ & 0 & 0
\end{tabular} \\
\hline \begin{tabular}{l|lll}
$c_{2}$ & $a_{21}$ & $a_{22}$ & $a_{23}$
\end{tabular} & \begin{tabular}{l|lll}
$c_{2}$ & $a_{21}$ & $a_{22}$ & 0
\end{tabular} \\
\hline \begin{tabular}{l|lll}
$c_{3}$ & $a_{31}$ & $a_{32}$ & $a_{33}$ \\
\end{tabular} & \begin{tabular}{l|lll}
$c_{3}$ & $a_{31}$ & $a_{32}$ & $a_{33}$ \\
\end{tabular} \\
\hline \begin{tabular}{l|lll} 
& $b_{1}$ & $b_{2}$ & $b_{3}$
\end{tabular} & $\begin{array}{lll}b_{1} & b_{2} & b_{3}\end{array}$ \\
\hline
\end{tabular}

Table 1 Butcher array for FIRK and DIRK subclasses of the three-stage IRK methods (see [7]) 
$I-h a_{i i} \frac{\partial f}{\partial y}$. It was reported in [8] that if all $a_{i i}$ are equal, the stored LU factorization of such a single matrix can be used repeatedly. This motivates a maximum of one LU decomposition in which the matrix $A$ is written as a product of a lower triangular matrix $L$ and an upper triangular matrix $U$. It means $A$ is decomposed as $A=L U$.

Drugs are administered into the body through several routes. The criteria for the selection of a delivery route are the patient's suitability, solubility of the drug, access to the disease's location, and the effectiveness in dealing with the specific disease. According to [12], intravenous, intramuscular, intranasal, intradermal/transdermal, and oral administration are the main drug delivery routes. Among the various delivery routes, oral delivery is the most widely used and commonly employed route of drug administration [12-16]. This is due to the potential advantages of high patient compliance [12, 14], ease of administration [12,15], and pain avoidance [16].

To facilitate a meaningful contribution to the pharmacokinetics field, numerous mathematical models for drug delivery systems have been developed, which can be found in [1723]. In [21], the established model described the mechanisms of how the human body handles nicardipine (NC)-cyclodextrin complexes injection in the gastrointestinal (GI) tract, distribution in plasma, and metabolism in the liver. Recently, [22] proposed a differential equation model to investigate the dynamic behavior drug resistance incorporating a delay in the process with conditions under which a Hopf bifurcation takes place, which leads to a periodic solution. The models in [23] outline the mechanisms of drug administration in the human body via oral and intravenous routes. To calculate the exact drug concentration in different compartments of blood and tissue medium, the Laplace transformation and eigenvalue method were applied. In this article, we solve the mathematical models of drug diffusion formulated by $[20,21,23]$ numerically. The models were formulated based on the diffusion process by applying Fick's principle and the law of mass action.

The stiffness phenomenon exhibited in the drug diffusion models needed to be treated using a suitable implicit numerical method. Backward differentiation formula (BDF) is the most popular class of implicit linear $k$-step methods for solving stiff IVPs. To overcome the drawback of the classical BDF method which approximates $y_{n+1}$ at $x_{n+1}$ one step at a time, seminal contributions have been made by [24]. The authors employed the block backward differentiation formula (BBDF) to accelerate the integration process. The BBDF produces a block of $y_{n+1}, y_{n+2}, \ldots, y_{n+k}$ approximations concurrently at each of the algorithms by using earlier blocks with each block containing $k$ points. Some authors have driven the further development of BBDF that proven to improve the performance in efficiency and accuracy upon solving stiff problems (refer to [25-28]). Although this approach is well established for stiff ODEs, the proof of stability properties has not been extensively studied. Therefore, it is sensible to contribute the relevant proof and modify existing BBDF methods to better accuracy in realizing the newly established scheme's full potential in approximating the solution of stiff pharmacokinetics models.

Dahlquist in [29] introduced the concept of $A$-stability to address the impact of stiffness on numerically solving IVPs (see [30]). The need for this desirable property, however, imposes a severe constraint on the option of suitable methods for stiff ODEs. This has, therefore, motivated us to design an efficient two-point block method based on BDF in a diagonally implicit manner that holds an $A$-stable property.

The rest of the paper is outlined as follows. In Sect. 2, we briefly illustrate the derivation of the proposed method. Section 3 provides the stability analysis and some relevant 
proofs. The mathematical models in which the method is applied are presented in Sect. 4. Section 5 is devoted to the discussion of the numerical results. Finally, Sect. 6 concludes this paper.

\section{$2 \rho$-Diagonally implicit block backward differentiation formula}

In this section, we derive the two-point $\rho$-diagonally implicit block backward differentiation formula ( $\rho$-DIBBDF) for the solution of the pharmacokinetics models developed by $[20,21,23]$. The general form of our method is based on the definition of linear multistep method (LMM) of BBDF as given by Ibrahim et al. in [24] in the form of

$$
\sum_{j=0}^{k+m-1} \alpha_{j-1, k} y_{n+j-1}=h_{n+m} \beta_{k+m-2, k}\left(f_{n+k}-\rho f_{n+k-1}\right)
$$

where $m=2$ is order of the method; $k=1,2$ for $y_{n+1}$ and $y_{n+2}$, respectively; $\rho$ is a free parameter; $h_{n+m}$ is step length; $\alpha_{k, k}=1 ; f_{n+k}=f\left(x_{n+k}, y_{n+k}\right)$ and $\beta_{k-1, k}=-\rho \beta_{k, k}$.

In the case of the LMM in (2), we associate the linear difference operator $\mathcal{L}$, defined by

$$
\mathcal{L}\left[y\left(x_{n}\right) ; h\right]=\sum_{j=0}^{k+m-1} \alpha_{j-1, k} y_{n+j-1}-h_{n+m} \beta_{k+m-2, k}\left(y_{n+k}^{\prime}-\rho y_{n+k-1}^{\prime}\right) .
$$

Expanding $y_{n+j-1}, y_{n+k}^{\prime}$ and $y_{n+k-1}^{\prime}$ as Taylor series about $x$ and collecting the terms in (3) gives

$$
\mathcal{L}\left[y\left(x_{n}\right) ; h\right]=C_{0} y(x)+C_{1} h_{n+m} y^{\prime}(x)+\cdots+C_{p} h_{n+m}^{p} y^{(p)}(x) .
$$

The constants $C_{p}$ are defined as

$$
\begin{aligned}
C_{0}= & \sum_{j=0}^{k+m-1} \alpha_{j-1, k}, \\
C_{1}= & \frac{(-s)^{p}}{p !} \alpha_{-1, k}+\sum_{j=2}^{k+m-1} \frac{(j-1)^{p}}{p !} \alpha_{j-1, k}-\frac{k^{(p-1)}}{(p-1) !} \beta_{k+m-2, k}+\rho \beta_{k+m-2, k}, \\
\vdots & \\
C_{p}= & \frac{(-s)^{p}}{p !} \alpha_{-1, k}+\sum_{j=2}^{k+m-1} \frac{(j-1)^{p}}{p !} \alpha_{j-1, k}-\frac{k^{(p-1)}}{(p-1) !} \beta_{k+m-2, k} \\
& +\frac{(k-1)^{(p-1)}}{(p-1) !} \rho \beta_{k+m-2, k}, \quad p \geq 2,
\end{aligned}
$$

where $s$ is the step length ratio $s=\frac{h_{n+m}}{h_{n+m-1}}$. The values of $k=1,2$ in (5) indicate the first and second point, respectively. Without losing the generality, it will be assumed from here on 
that $\alpha_{1,1}=1, \alpha_{2,2}=1$, and $\alpha_{0,2}=0$. Equating the results term by term with (4) yields

$$
\begin{aligned}
& \alpha_{-1, k}=\left[\begin{array}{c}
-\frac{(\rho+1)}{s(s \rho-s-2)} \\
-\frac{\rho+1}{(s+1)[(s+1) \rho-(s+3)]}
\end{array}\right], \quad \alpha_{0, k}=\left[\begin{array}{c}
-\frac{(s+1)[(s-1) \rho-(s+1)]}{s(s \rho-s-2)} \\
0
\end{array}\right], \\
& \alpha_{1, k}=\left[\begin{array}{c}
1 \\
-\frac{(s+2)(s \rho-s-2)}{(s+1)[(s+1) \rho-(s+3)]}
\end{array}\right], \quad \alpha_{2, k}=\left[\begin{array}{l}
0 \\
1
\end{array}\right], \quad \beta_{-1, k}=\left[\begin{array}{l}
0 \\
0
\end{array}\right], \\
& \beta_{0, k}=\left[\begin{array}{c}
-\frac{s+1}{s(s \rho-s-2)} \\
0
\end{array}\right], \quad \beta_{1, k}=\left[\begin{array}{c}
\frac{s+1}{s(s \rho-s-2)} \\
\frac{s+2}{(s+1) \rho-(s+3)}
\end{array}\right], \quad \beta_{2, k}=\left[\begin{array}{c}
0 \\
-\frac{s+2}{(s+1) \rho-(s+3)}
\end{array}\right] .
\end{aligned}
$$

Next, the coefficients of $\alpha_{j-1, k}$ and $\beta_{k+m-2, k}$ obtained in (6) are substituted into (2), and by considering $h_{n+m}=h_{n+m-1}$ for all $n$, we have $s=1$ in (5), which gives the following corrector formula for the two-point $\rho$-DIBBDF in constant step length:

$$
\begin{aligned}
{\left[\begin{array}{cc}
1 & 0 \\
-\frac{3}{4}\left(\frac{\rho-3}{\rho-2}\right) & 1
\end{array}\right]\left[\begin{array}{l}
y_{n+1} \\
y_{n+2}
\end{array}\right]=} & {\left[\begin{array}{cc}
\frac{\rho+1}{\rho-3} & -\frac{4}{\rho-3} \\
\frac{1}{4}\left(\frac{\rho+1}{\rho-2}\right) & 0
\end{array}\right]\left[\begin{array}{c}
y_{n-1} \\
y_{n}
\end{array}\right]+h\left[\begin{array}{cc}
0 & \frac{2 \rho}{\rho-3} \\
0 & 0
\end{array}\right]\left[\begin{array}{c}
f_{n-1} \\
f_{n}
\end{array}\right] } \\
& +h\left[\begin{array}{cc}
-\frac{2}{\rho-3} & 0 \\
\frac{3 \rho}{2 \rho-4} & -\frac{3}{2 \rho-4}
\end{array}\right]\left[\begin{array}{l}
f_{n+1} \\
f_{n+2}
\end{array}\right] .
\end{aligned}
$$

\section{Stability analysis}

In this section, the stability properties of $\rho$-DIBBDF will be discussed for $\rho \in(-1,1)$. The parameter $\rho$ is restricted to $(-1,1)$ so that the underlying formula in (2) satisfies the necessary condition for stiff stability. The relevant proof for $k=1$ provided in this section is referred to the theorem by [31] (see [32]). The approach adopted to prove some stability properties for $k=2$ is similar to that of $k=1$.

As the first consideration, let us define $\varrho(\xi)$ and $\sigma(\xi)$ as the first and second characteristic polynomials, respectively, in the following terms:

$$
\begin{aligned}
& \varrho(\xi)=\sum_{j=0}^{m} \alpha_{j-1, k} \xi^{j}, \\
& \sigma(\xi)=\sum_{j=0}^{m} \beta_{j-1, k} \xi^{j},
\end{aligned}
$$

which are equivalent to $\varrho(\xi)=\alpha_{1,1} \xi^{2}+\alpha_{0,1} \xi+\alpha_{-1,1}$ and $\sigma(\xi)=\beta_{1,1}\left(\xi^{2}-\rho \xi\right)$. For this study, it is of interest to investigate the stability features of the method by using the Möbius transformation $\xi=\frac{1+z}{1-z}$. It maps the unit disc $\{\xi \in \mathbb{C}|| \xi \mid<1\}$ and the unit circle onto the negative half-plane and the imaginary axis, respectively. Given that

$$
\begin{aligned}
& a(z)=\left(\frac{1-z}{2}\right)^{2} \varrho\left(\frac{1+z}{1-z}\right), \\
& b(z)=\left(\frac{1-z}{2}\right)^{2} \sigma\left(\frac{1+z}{1-z}\right),
\end{aligned}
$$


are the two polynomials in $z$ to define $Q(z, \mu)=a(z)-\mu b(z)$ where $\mu=h \lambda$, these polynomials can be expressed as

$$
\begin{aligned}
& 4 a(z)=2\left(1+\alpha_{-1,1}\right) z^{2}-2\left(-1+\alpha_{-1,1}\right) z, \\
& 4 b(z)=\beta_{1,1}\left[(1+\rho) z^{2}+2 z+(1-\rho)\right],
\end{aligned}
$$

which imply that $4 Q(z, \mu)=q_{2} z^{2}+q_{1} z+q_{0}$. The coefficients of $4 Q(z, \mu)$ are given by

$$
\begin{aligned}
& q_{2}=2\left(1+\alpha_{-1,1}\right)-\beta_{1,1}(1+\rho) \mu, \\
& q_{1}=2\left(1-\alpha_{-1,1}\right)-2 \mu, \\
& q_{0}=\beta_{1,1}(1-\rho) \mu .
\end{aligned}
$$

From (8), the polynomial $\pi(\xi, \mu)=\varrho(\xi)-\mu \sigma(\xi)$ is formed. It leads to some definitions, as described below.

Definition 1 The region of absolute stability for (7) is given by $\tilde{A}=\left\{\mu \in \mathbb{C}|| \xi_{i}(\mu) \mid<1, i=\right.$ $1,2, \ldots, m\}$.

Definition 2 The method in (7) is $A_{0}$-stable if $\{\mu \in \mathbb{C} \mid \operatorname{Im}(\mu)=0,-\infty<\mu<0\} \subset \tilde{A}$.

The following lemma will be used in the next theorem.

Lemma 1 Assume $q(x)=q_{2} x^{2}+q_{1} x+q_{0}$, where $q_{2}, q_{1}, q_{0}$ are real values and $q_{2} \neq 0$, then $q(x)$ is a Hurwitz polynomial if and only if all $a_{i}$ s are either positive or negative.

Proof See [33].

As outlined in [32], a method is said to be $A_{0}$-stable if and only if its corresponding $Q(z, \mu)$ is a Hurwitz polynomial for all $\mu<0$.

Theorem 1 The method is $A_{0}$-stable for all $\rho \in(-1,1)$.

Proof Since $\alpha_{-1,1}, \beta_{1,1}>0$ for all $\rho$ in the interval $(-1,1)$, then we have $q_{0}, q_{2}>0$. Now, $q_{1}$ will be greater than 0 if and only if $\left(1-\alpha_{-1,1}\right) \geq 0$. Thus, by Lemma $1, Q(z, \mu)$ is a Hurwitz polynomial for all $\mu<0$ if and only if $\left(1-\alpha_{-1,1}\right) \geq 0$.

Definition 3 The formula in (7) is said to be $A$-stable if $\{\mu \in \mathbb{C} \mid \operatorname{Re}(\mu)<0\} \subset \tilde{A}$ (refer to $[34])$.

Lemma 2 Let $g(x)=a x^{2}+b x+c$, where $a, b$, c are real values and $a>0$. Assume also $g(-1)>0$ and $g(1)=0$. Then $g(x) \geq 0$ for $-1 \leq x \leq 1$ if and only if $2 a+b \leq 0$.

Proof Given that $a>0$, then the graph of $g(x)$ will concave upward and the vertex is $x_{0}=$ $\frac{-b}{2 a}$. Since $g(1)=0$, it means that 1 is the $x$-intercept of $g(x)$ and $g(-1)>0$, and we need to have $x_{0}>-1$. Now, suppose $2 a+b \leq 0$; if $x \leq 1$, then $x_{0} \geq-1$ implies that $g(x) \geq 0$. Next, we assume $g(x) \geq 0$ for $-1 \leq x \leq 1$. If $-1<x_{0}<1$, then $g\left(x_{0}\right)<g(1)$. However, we have $g(1)=0$; thus, $x_{0} \geq 1$. 
Theorem 2 Suppose all the roots of $\sigma(\xi)$ in (8) have the modulus of less than 1 . Then the method in (2) is A-stable if and only if

$$
P_{m}(\xi)=\sum_{j=0}^{m} \gamma_{j} T_{j}(\xi) \geq 0 \quad \text { for }-1 \leq \xi \leq 1,
$$

where

$$
\begin{aligned}
& \gamma_{0}=\sum_{i=0}^{m} \alpha_{i-1, k} \beta_{i-1, k}, \\
& \gamma_{j}=\sum_{i=0}^{m-j}\left(\alpha_{i+j-1, k} \beta_{i-1, k}+\alpha_{i-1, k} \beta_{i+j-1, k}\right), \quad 1 \leq j \leq m,
\end{aligned}
$$

and $T_{j}(\xi)$ is the jth Chebyshev polynomial.

Proof By expanding $\gamma_{0}$ and $\gamma_{j}$, taking $\beta_{0,1}=-\rho \beta_{1,1}$ and $\alpha_{-1,1}+\alpha_{0,1}+1=0$ as in (5), we have

$$
\begin{aligned}
\gamma_{0} & =\alpha_{-1,1} \beta_{-1,1}+\alpha_{0,1} \beta_{0,1}+\alpha_{1,1} \beta_{1,1} \\
& =\beta_{1,1}\left(1-\rho \alpha_{0,1}\right), \\
\gamma_{1} & =\left(\alpha_{0,1} \beta_{-1,1}+\alpha_{-1,1} \beta_{0,1}\right)+\left(\alpha_{1,1} \beta_{0,1}+\alpha_{0,1} \beta_{1,1}\right) \\
& =\beta_{1,1} \alpha_{0,1}(1+\rho), \\
\gamma_{2} & =\alpha_{1,1} \beta_{-1,1}+\alpha_{-1,1} \beta_{1,1} \\
& =-\beta_{1,1}\left(1+\alpha_{0,1}\right) .
\end{aligned}
$$

Let $T_{0}(\xi)=1, T_{1}(\xi)=\xi$, and $T_{2}(\xi)=2 \xi^{2}-1$ be the Chebyshev polynomial of degree 0,1 , and 2, respectively; then we define $P_{m}(\xi)=\sum_{j=0}^{m} \gamma_{j} T_{j}(\xi)$ which is equivalent to

$$
\begin{aligned}
P_{2}(\xi) & =\gamma_{0} T_{0}(\xi)+\gamma_{1} T_{1}(\xi)+\gamma_{2} T_{2}(\xi) \\
& =\beta_{1,1}\left(1-\rho \alpha_{0,1}\right)+\beta_{1,1} \alpha_{0,1}(1+\rho) \xi-\beta_{1,1}\left(1+\alpha_{0,1}\right)\left(2 \xi^{2}-1\right) \\
& =\beta_{1,1}\left[1-\rho \alpha_{0,1}+\alpha_{0,1}(1+\rho) \xi-\left(\alpha_{0,1}+1\right)\left(2 \xi^{2}-1\right)\right] \\
& =\beta_{1,1}\left[2 \alpha_{-1,1} \xi^{2}+\alpha_{0,1}(1+\rho) \xi+1-\rho \alpha_{0,1}-\alpha_{-1,1}\right] \\
& =\beta_{1,1}\left[2 \alpha_{-1,1} \xi^{2}+\alpha_{0,1}(1+\rho) \xi+2+\alpha_{0,1}(1-\rho)\right] .
\end{aligned}
$$

Now, by computing $P_{2}(1)$ and $P_{2}(-1)$, we obtain

$$
\begin{aligned}
P_{2}(1) & =\beta_{1,1}\left[2 \alpha_{-1,1}+2 \alpha_{0,1}+2\right] \\
& =0, \\
P_{2}(-1) & =\beta_{1,1}\left[2 \alpha_{-1,1}-\alpha_{0,1}(1+\rho)+2+\alpha_{0,1}(1-\rho)\right] \\
& =-2 \beta_{1,1} \alpha_{0,1}(1+\rho) .
\end{aligned}
$$

Since $\beta_{1,1}>0$ and $\alpha_{0,1}<0$ for all $\rho \in(-1,1)$, then we have $P_{2}(-1)>0$. Thus, by Lemma 2, $P_{2}(\xi) \geq 0$ for $-1 \leq \xi \leq 1$ if and only if $2 a+b \leq 0$. By substituting the coefficients of $\xi^{2}$ and 
$\xi$ in $P_{2}(\xi)$, we obtain $2 a+b=4 \alpha_{-1,1}+\alpha_{0,1}(1+\rho)$, which can be simplified as

$$
4 \alpha_{-1,1}+\alpha_{0,1}(1+\rho)=\frac{(s-1)(1+\rho)[(3-\rho) s+(1-\rho)]}{1-\rho+2 s},
$$

where $1+\rho>0,1-\rho+2 s>0$, and $(3-\rho) s+(1-\rho)>0$ for all $s>0$ and $\rho \in(-1,1)$. Thus, $4 \alpha_{-1,1}+\alpha_{0,1}(1+\rho) \leq 0$ if and only if $s-1 \leq 0$, implying that $s \leq 1$.

Corollary 1 The method is A-stable for all $\rho \in(-1,1)$.

Proof Since this theorem satisfies $s=1$, i.e., the constant step length formula, then the underlying formula in (7) is $A$-stable for all $\rho$ in the interval $(-1,1)$.

To determine whether the numerical method with the chosen $\rho$ is capable of delivering acceptable results, the stability of the method needs to be investigated. Some desirable stability criteria for a numerical method are discussed here, namely zero stability, absolute stability, and convergence. Later, the restrictive requirement on the step length will be studied. For absolute stability purposes, the free parameter $\rho$ is chosen in which it is restricted to $(-1,1)$ (see [25]). Here, we select $\rho=-\frac{3}{4}$. A detailed discussion for this selection of $\rho$ can be found in [35].

\subsection{Zero and absolute stability}

Denoting $h \lambda$ by $\hat{h}$, we have the following definitions pertinent to stiffness.

Definition 4 The method in (7) is zero stable if its characteristic polynomial has a simple root at +1 and all the remaining roots lie strictly within the unit circle (refer to [34]).

Definition 5 The method in (7) is $A$-stable if $\Re_{A} \supseteq\{\hat{h} \mid \operatorname{Re}(\hat{h})<0\}$, where $\Re_{A}$ is the region of absolute stability for the method (refer to [5]).

Applying the Dahlquist test equation $y^{\prime}=f(x, y)=\lambda y$ and $h \lambda=\hat{h}$ into (7) yields

$$
\left[\begin{array}{cc}
1+\frac{2}{\rho-3} \hat{h} & 0 \\
-\frac{3}{4}\left(\frac{\rho-3}{\rho-2}\right)-\frac{3 \rho}{2 \rho-4} \hat{h} & 1+\frac{3}{2 \rho-4} \hat{h}
\end{array}\right]\left[\begin{array}{l}
y_{n+1} \\
y_{n+2}
\end{array}\right]=\left[\begin{array}{cc}
\frac{\rho+1}{\rho-3} & -\frac{4}{\rho-3}+\frac{2 \rho}{\rho-3} \hat{h} \\
\frac{1}{4}\left(\frac{\rho+1}{\rho-2}\right) & 0
\end{array}\right]\left[\begin{array}{c}
y_{n-1} \\
y_{n}
\end{array}\right],
$$

which may be written as $A Y_{m}=B Y_{m-1}$. The first characteristic polynomial of our method can be constructed in terms of its roots $r$ by evaluating $\operatorname{det}(A r-B)$. The stability polynomial is given as follows:

$$
\begin{aligned}
\operatorname{SP}(\hat{h})= & {\left[\frac{-1}{2(\rho-3)(\rho-2)}\right] 6 \hat{h}^{2} \rho^{2} r-6 \hat{h}^{2} r^{2}+3 \hat{h} \rho^{2} r-7 \hat{h} \rho r^{2}-2 \rho^{2} r^{2}+\hat{h} \rho^{2} } \\
& -18 \hat{h} \rho r+17 \hat{h} r^{2}+2 \rho^{2} r+10 \rho r^{2}+\hat{h} \rho+3 \hat{h} r-8 \rho r-12 r^{2}-2 \rho+14 r-2 \\
= & 0 .
\end{aligned}
$$

By setting $\rho=-\frac{3}{4}, \hat{h}=0$ and solving (10) with respect to $r$, it yields the roots, $r=0.01122$ and $r=1$. Thus, by Definition 4, we conclude that the method is zero stable.

Next, the boundary of the stability region is determined. The boundary locus technique is the most convenient method for finding the region of absolute stability. The region of 
Figure 1 Stability region of $\rho$-DIBBDF for $\rho=-\frac{3}{4}$

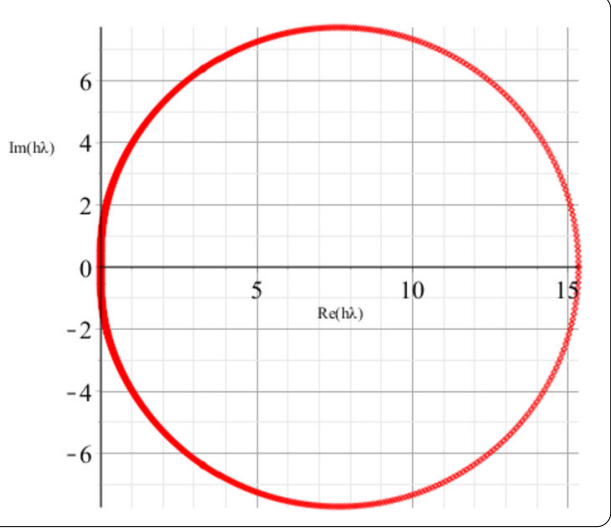

absolute stability for a range of $\theta \in[0,2 \pi]$ is obtained by setting $r=e^{i \theta}$ for which $|r| \leq 1$. As depicted in Fig. 1, the stable region lies outside the closed contour of the graph. Thus, by Definition 5 , the method is considered $A$-stable.

\subsection{Error constant}

Definition 6 LMM (2) and the associated difference operator $L$ defined by (3) are said to be of order $p$ if in (5), $C_{0}=C_{1}=\cdots=C_{p}=0, C_{p+1} \neq 0$, where $C_{p+1}$ is the error constant (see $[3])$.

For $\rho=-\frac{3}{4}$, by substituting the coefficients of $\alpha_{j-1, k}$ and $\beta_{k+m-2, k}$ into (5), we obtained $C_{0}=C_{1}=C_{2}=0$ and $C_{3}=\left[-\frac{1}{9}-\frac{3}{22}\right]^{T}$. Thus, the method is of order 2 with $C_{3}$ being the error constant.

\subsection{Convergence}

The basic feature that requires an acceptable LMM is that the numerical solution converges to the exact solution $y(x)$ as $h$ approaches zero. The following theorem, the proof of which can be found in [36], sets out the conditions on $f(x, y)$ which guarantee the existence of a unique solution of the IVP in (1) (as in [3]).

Theorem 3 Let $f(x, y)$ be defined and continuous for all points $(x, y)$ in the region $D$ defined by $a \leq x \leq b,-\infty<y<\infty, a$ and $b$ finite, and let there exist a constant $L$ as a Lipschitz constant such that, for every $x, y, y^{*}$ such that $(x, y)$ and $\left(x, y^{*}\right)$ are both in $D$,

$$
\left|f(x, y)-f\left(x, y^{*}\right)\right| \leq L\left|y-y^{*}\right|
$$

Then, if $y(a)=\beta$ is any given number, there exists a unique solution $y(x)$ of the IVP in (1), where $y(x)$ is continuous and differentiable for all $(x, y)$ in $D$ (refer to [3]).

Consequently, this leads to the definition of convergence as given below.

Definition 7 The LMM in (2) is said to be convergent if, for all IVPs in (1) subject to the hypotheses of Theorem 3, we have that

$$
\lim _{h \rightarrow 0} y_{n}=y\left(x_{n}\right)
$$

holds for all $x \in[a, b]$ and for all solutions $\left\{y_{n}\right\}$, where $n h=x-a$ (refer to [3]). 
From (7), we have the formula for the two-point $\rho$-DIBBDF written for the approximate solutions as follows:

$$
\begin{aligned}
& y_{n+1}=-\frac{1}{15} y_{n-1}+\frac{16}{15} y_{n}+\frac{2}{5} h f\left(x_{n}, y_{n}\right)+\frac{8}{15} h f\left(x_{n+1}, y_{n+1}\right), \\
& y_{n+2}=-\frac{1}{44} y_{n-1}+\frac{45}{44} y_{n+1}+\frac{9}{22} h f\left(x_{n+1}, y_{n+1}\right)+\frac{6}{11} h f\left(x_{n+2}, y_{n+2}\right),
\end{aligned}
$$

and for the exact solutions as follows:

$$
\begin{aligned}
Y_{n+1}= & -\frac{1}{15} Y_{n-1}+\frac{16}{15} Y_{n}+\frac{2}{5} h f\left(x_{n}, Y_{n}\right)+\frac{8}{15} h f\left(x_{n+1}, Y_{n+1}\right)-\frac{1}{9} h^{3} Y^{(3)}\left(\xi_{n}\right), \\
Y_{n+2}= & -\frac{1}{44} Y_{n-1}+\frac{45}{44} Y_{n+1}+\frac{9}{22} h f\left(x_{n+1}, Y_{n+1}\right)+\frac{6}{11} h f\left(x_{n+2}, Y_{n+2}\right) \\
& -\frac{3}{22} h^{3} Y^{(3)}\left(\xi_{n}\right) .
\end{aligned}
$$

Given that

$$
\lim _{h \rightarrow 0} y_{n+1}=Y_{n+1}, \quad \lim _{h \rightarrow 0} y_{n+2}=Y_{n+2}
$$

are the conditions for the convergence of the approximate solutions. Subtracting (13) from (14) yields

$$
\begin{aligned}
Y_{n+1}-y_{n+1}= & -\frac{1}{15}\left[Y_{n-1}-y_{n-1}\right]+\frac{16}{15}\left[Y_{n}-y_{n}\right] \\
& +\frac{2}{5} h\left[f\left(x_{n}, Y_{n}\right)-f\left(x_{n}, y_{n}\right)\right]+\frac{8}{15} h\left[f\left(x_{n+1}, Y_{n+1}\right)-f\left(x_{n+1}, y_{n+1}\right)\right] \\
& -\frac{1}{9} h^{3} Y^{(3)}\left(\xi_{n}\right), \\
Y_{n+2}-y_{n+2}= & -\frac{1}{44}\left[Y_{n-1}-y_{n-1}\right]+\frac{45}{44}\left[Y_{n+1}-y_{n+1}\right] \\
& +\frac{9}{22} h\left[f\left(x_{n+1}, Y_{n+1}\right)-f\left(x_{n+1}, y_{n+1}\right)\right] \\
& +\frac{6}{11} h\left[f\left(x_{n+2}, Y_{n+2}\right)-f\left(x_{n+2}, y_{n+2}\right)\right]-\frac{3}{22} h^{3} Y^{(3)}\left(\xi_{n}\right) .
\end{aligned}
$$

By denoting $Y_{n+r}-y_{n+r}=d_{n+r}$, where $r=-1,0,1,2$, then (16) becomes

$$
\begin{aligned}
& \left|d_{n+1}\right| \leq-\frac{1}{15}\left|d_{n-1}\right|+\left(\frac{16}{15}+\frac{2}{5} h L\right)\left|d_{n}\right|+\frac{8}{15} h L\left|d_{n+1}\right|-\frac{1}{9} h^{3} Q \\
& \left|d_{n+2}\right| \leq-\frac{1}{44}\left|d_{n-1}\right|+\left(\frac{45}{44}+\frac{9}{22} h L\right)\left|d_{n+1}\right|+\frac{6}{11} h L\left|d_{n+2}\right|-\frac{3}{22} h^{3} Q
\end{aligned}
$$

where $Q=\underbrace{\max }_{a \leq x \leq b}\left|Y^{(3)}(x)\right|$. As $h$ approaches zero, then $\left|d_{n+k}\right| \leq\left|d_{n}\right|$ which implies that $Y_{n+k}-Y_{n} \leq y_{n+k}-y_{n}$ for $k=1,2$. Since the condition in (15) is satisfied, we conclude that the proposed method converges. 


\subsection{Step length restriction}

To deal with the stability phenomenon as mentioned previously in Sect. 1, we discuss the following definitions given by [5]. The step length restriction is discussed in a similar way as in [37].

Definition 8 The LMM in (2) is said to be absolutely stable for a given $\hat{h}$ if all roots of $\pi(r, \hat{h})$ satisfy $\left|r_{t}\right|<1, t=1,2, \ldots, k$; otherwise, the method is said to be absolutely unstable.

Definition 9 The LMM in (2) is said to have a region of absolute stability $\Re_{A}$, where $\mathfrak{R}_{A}$ is a region of the complex $\hat{h}$-plane, if it is absolutely stable for all $\hat{h} \in \Re_{A}$. The intersection of $\Re_{A}$ with the real axis is called the interval of absolute stability.

From Fig. 1, one could observe that $\rho$-DIBBDF is absolutely stable except when $\hat{h} \in$ $(0,15.333)$. This interval is known as the interval of unstable region. As described in Definitions 8 and 9 , the $\Re_{A}$ region is defined by the requirement that all the roots of $\operatorname{SP}(\hat{h})$ have a module of less than 1 for all $\hat{h} \in \Re_{A}$. For the method to be stable, $h$ must lie within a certain range. Thus, we are motivated to find the value of $h \lambda$ so that $|r|<1$. By substituting the endpoint of the interval into $\operatorname{SP}(\hat{h})$ in $(10)$, we obtain

$$
\varphi=52.85193134 \hat{h}^{2}-53.01621516 \hat{h}+0.1636333333 .
$$

On solving (18), we choose the value of $|\hat{h}|<1$. Therefore, the step length is restricted to $|H|<0.003096032803$ which is equivalent to

$$
h<\left|\frac{0.003096032803}{\lambda}\right| \text {. }
$$

Let us consider a stiff problem with $\lambda=-100$, then we will have

$$
h<\left|\frac{0.003096032803}{-100}\right| \text {, }
$$

which indicates that absolute stability is achieved if the step length chosen is $h<$ $3.096032803 \times 10^{-5}$.

\section{Mathematical model}

In this section, we approximate the numerical solutions for five examples of pharmacokinetics models from various literature. A principle based on the law of conservation of mass is applied in the formulation of the mathematical models. It is known as balance law and can be discovered in [38]. Let $c(t)$ denote the concentration of drug in the compartment at time $t$ (hour), hence the balance law applied is

$$
\frac{d c}{d t}=\text { input rate of drug }- \text { output rate of drug, }
$$

where $\frac{d c}{d t}$ is the rate of change for $c(t)$. 


\subsection{Model A}

In 1970, in the works of [17], a linear, two-compartment, and open model for drug distribution was presented. Then, a two-compartment pharmacokinetic model was developed by [19] to model the flow of drugs through the body compartments: the GI tract and the circulatory system. The drug is taken orally on a regular basis, resulting in a dosage pulse delivered to one compartment (GI tract). From that point, the drug moves into another compartment (bloodstream) at a rate relative to its concentration in the first compartment. Finally, the drug is metabolized and removed from the blood at a rate corresponding to its concentration there (refer to [20]).

Consider the following model consisting of the concentration of drug in the GI tract $c_{1}(t)$ and its concentration in the bloodstream $c_{2}(t)$ as a function of time $t$ :

$$
\begin{aligned}
& \frac{d c_{1}(t)}{d t}=-a c_{1}(t)+D ; \quad c_{1}(0)=1, \\
& \frac{d c_{2}(t)}{d t}=a c_{1}(t)-b c_{2}(t) ; \quad c_{2}(0)=0,
\end{aligned}
$$

where $D$ is the drug intake regimen. Noting that $a=2 \ln (2)$ and $b=\frac{\ln (2)}{5}$, the solution of differential equations in (20) for $0 \leq t \leq 6$ are given by

$$
\begin{aligned}
& c_{1}(t)=e^{-a t}, \\
& c_{2}(t)=-\frac{a}{a-b}\left(e^{-a t}-e^{-b t}\right), \quad a \neq b .
\end{aligned}
$$

\subsection{Model B}

The formulation of drugs with different drug carrier materials is applied widely in the pharmaceutical sciences field to control drug delivery and improve drug release. The authors in [21] had formulated the NC delivery with cyclodextrin (CD) complexes as excipients. The formulation of the NC with hydroxypropyl- $\beta$-cyclodextrin $(N C / H P \beta C D)$ and triacetyl- $\beta$-cyclodextrin $(N C / T A \beta C D)$ modeled in Fig. 2 , where $k_{G I}$ and $k_{\text {Plasma }}$ are the rate constants in the GI tract and plasma, respectively.

The rate equations corresponding to the scheme in Fig. 2 are

$$
\begin{aligned}
& \frac{d C_{A}(t)}{d t}=-k_{G I} C_{A}(t) ; \quad C_{A}(0)=1.0 \mathrm{ng} / \mathrm{mL}, \\
& \frac{d C_{B}(t)}{d t}=k_{G I} C_{A}(t)-k_{\text {Plasma }} C_{B}(t) ; \quad C_{B}(0)=0,
\end{aligned}
$$

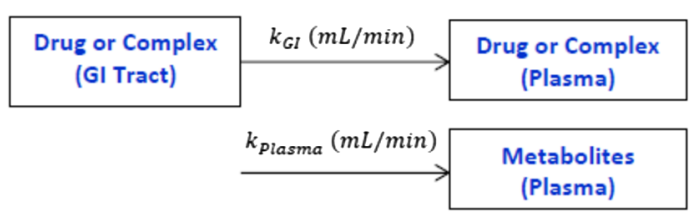

Figure 2 Mechanism of irreversible series of the NC delivery in Model B (see [21]) 
where $C_{A}$ and $C_{B}$ are the concentrations in the GI tract and plasma, respectively, and $t \in[0,25]$. On solving (22), we have

$$
\begin{aligned}
& C_{A}(t)=e^{-k_{G I} t}, \\
& C_{B}(t)=-\frac{k_{G I}}{k_{G I}-k_{\text {Plasma }}}\left(e^{-k_{G I} t}-e^{-k_{\text {Plasma }} t}\right), \quad k_{G I} \neq k_{\text {Plasma }} .
\end{aligned}
$$

The refined rate constants calculated from the predicted (GI) and experimental (Plasma) are listed in Table 2 as follows.

\subsection{Model C}

In this subsection, three mathematical models for drug administration through oral and intravenous routes in the human body are presented. The models have been formulated in the form of IVPs by [23]. The values of the rate constants for the diffusion models are taken from [23] and the eigenvalues $\lambda$ computed on Maple are listed in Table 3.

\subsubsection{Model C(i)}

The first model included two compartments of the GI tract and bloodstream, which illustrated the oral drug administration shown in Fig. 3.

Table 2 Model parameter values in Model B (see [21])

\begin{tabular}{lll}
\hline Compound & $k_{G l}(\mathrm{~mL} / \mathrm{min})$ & $k_{\text {Plasma }}(\mathrm{mL} / \mathrm{min})$ \\
\hline (i) NC & 3.18 & 0.99 \\
(ii) NC/HP $\beta C D$ & 0.59 & 0.43 \\
(iii) NC/TA $\beta C D$ & 1.00 & 0.29 \\
\hline
\end{tabular}

Table 3 Model parameter values in Models C(i), (ii), and (iii) for $t \in[0,6]$

\begin{tabular}{llll}
\hline Model & Parameter & Description & Value \\
\hline Model C(i) & $c_{0}$ & Initial concentration of drug dosage & 500 units $^{-1}$ \\
& $k_{1}$ & Rate constant of drug in Gl tract & 0.9776 hour $^{-1}$ \\
& $k_{c}$ & Clearance constant & 0.2213 hour $^{-1}$ \\
& $\lambda$ & Eigenvalues & $-0.7448,-0.9776$ \\
Model C(ii) & $c_{0}$ & Initial concentration of drug dosage & 500 units $^{-1}$ \\
& $k_{b}$ & Rate constant of drug in bloodstream & 0.9776 hour $^{-1}$ \\
& $k_{t}$ & Rate constant of drug in tissue medium & 0.3293 hour $^{-1}$ \\
& $k_{c}$ & Clearance constant & 0.2213 hour $^{-1}$ \\
& $\lambda$ & Eigenvalues & $-0.0492,-1.4789$ \\
Model C(iii) & $c_{0}$ & Initial concentration of drug dosage & 500 units $^{-1}$ \\
& $k_{a b}$ & Rate constant of drug in arterial blood & 0.9776 hour $^{-1}$ \\
& $k_{t}$ & Rate constant of drug in tissue medium & 0.3293 hour $^{-1}$ \\
& $k_{c}$ & Clearance constant & 0.2213 hour $^{-1}$ \\
& $\lambda$ & Eigenvalues & $-0.2213,-0.3293,-0.9776$ \\
\hline
\end{tabular}

Figure 3 Mechanism of oral drug administration in Model C(i) (refer to [23])

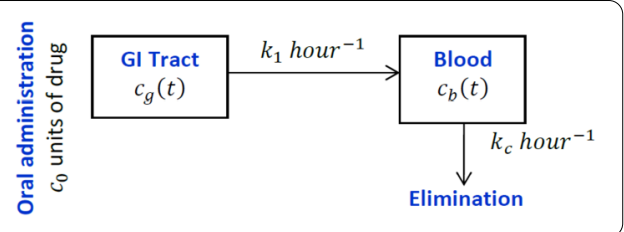


The two-compartment mathematical model in Fig. 3 is presented in a linear ODEs approach as follows:

$$
\begin{aligned}
& \frac{d c_{g}(t)}{d t}=-k_{1} c_{g}(t) ; \quad c_{g}(0)=c_{0}, \\
& \frac{d c_{b}(t)}{d t}=k_{1} c_{g}(t)-k_{c} c_{b}(t) ; \quad c_{b}(0)=0 .
\end{aligned}
$$

The drug delivery model in (1) is presented in terms of its concentration in two compartments: the first compartment in the GI tract $c_{g}(t)$ and the second compartment in blood $c_{b}(t) . c_{0}$ denotes the initial concentration of drug dosage, while $k_{1}$ and $k_{c}$ indicate the inter-compartment rate constant and clearance constant, respectively, where $k_{1}, k_{c}>0$. By solving equation (24), we have

$$
\begin{aligned}
& c_{g}(t)=c_{0} e^{-k_{1} t}, \\
& c_{b}(t)=\frac{c_{0} k_{1}}{k_{1}-k_{c}}\left(e^{-k_{c} t}-e^{-k_{1} t}\right), \quad k_{1} \neq k_{c} .
\end{aligned}
$$

\subsubsection{Model C(ii)}

For this intravenous drug model, two compartments are involved viz. blood and tissue as the main exchangers. The drug is injected into the bloodstream and transmitted to the tissue where the drug has a therapeutic effect. A schematic presentation of the intravenous drug administration based on reversible and irreversible rate constants is shown in Fig. 4.

The mathematical formulation of this two-compartment model can be expressed by the following ODEs:

$$
\begin{aligned}
& \frac{d c_{b}(t)}{d t}=-\left(k_{b}+k_{c}\right) c_{b}(t)+k_{t} c_{t}(t) ; \quad c_{b}(0)=c_{0}, \\
& \frac{d c_{t}(t)}{d t}=k_{b} c_{b}(t)-k_{t} c_{t}(t) ; \quad c_{t}(0)=0,
\end{aligned}
$$

in which $k_{b}$ and $k_{t}$ are the rate constants for the blood and tissue, respectively, with $c_{0}$ referring to the initial drug dosage and $k_{c}$ denoting the clearance rate. By applying Laplace transformation (see [23]), the resulting differential equations of (26) are solved, yielding

$$
\begin{aligned}
& c_{b}(t)=\frac{c_{0}}{\xi_{2}-\xi_{1}}\left[\left(-\xi_{1}+k_{t}\right) e^{-\xi_{1} t}-\left(-\xi_{2}+k_{t}\right) e^{-\xi_{2} t}\right], \\
& c_{t}(t)=\frac{c_{0} k_{b}}{\xi_{1}-\xi_{2}}\left[e^{-\xi_{1} t}-e^{-\xi_{2} t}\right],
\end{aligned}
$$

$$
\begin{aligned}
& \text { Figure } 4 \text { Mechanism of intravenous drug } \\
& \text { administration in Model C(ii) (refer to [23]) }
\end{aligned}
$$

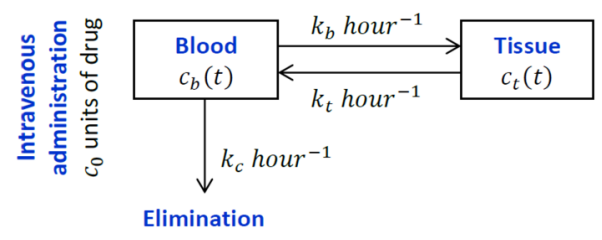




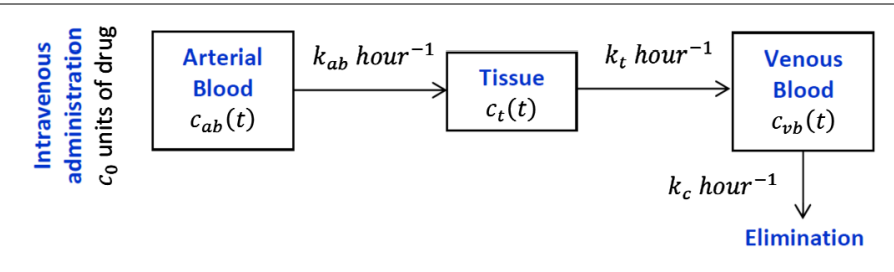

Figure 5 Mechanism of intravenous drug administration in Model C(iii) (refer to [23])

where $-\xi_{i}$ are real eigenvalues and are given by

$$
\xi_{i}=\frac{1}{2}\left[-\left(k_{b}+k_{t}+k_{c}\right) \pm \sqrt{\left(k_{b}+k_{t}+k_{c}\right)^{2}-4 k_{c} k_{t}}\right], \quad i=1,2
$$

\subsubsection{Model C(iii)}

The delivery mechanism of Model C(iii) is a one-directional model which is presented in terms of drug concentrations in the following compartments: arterial blood $c_{a b}(t)$; tissue $c_{t}(t)$; and venous blood $c_{v b}(t)$. The drug administration through the three compartments has the schematic view as illustrated in Fig. 5.

The scheme modeled in Fig. 5 is a pictorial presentation of the following system of ODEs:

$$
\begin{array}{ll}
\frac{d c_{a b}(t)}{d t}=-k_{a b} c_{a b}(t) ; & c_{a b}(0)=c_{0}, \\
\frac{d c_{t}(t)}{d t}=k_{a b} c_{a b}(t)-k_{t} c_{t}(t) ; & c_{t}(0)=0, \\
\frac{d c_{v b}(t)}{d t}=k_{t} c_{t}(t)-k_{c} c_{v b}(t) ; & c_{v b}(0)=0 .
\end{array}
$$

The flow rate of the drug from the arterial blood to the tissue compartment is represented by $k_{a b}$ and from the tissue compartment to the venous blood by $k_{t}$, while the rate of elimination is denoted by $k_{c}$. As presented in [23], the analytical solution of (28) is obtained as follows:

$$
\begin{aligned}
& c_{a b}(t)=c_{0} e^{-k_{a b} t} \\
& c_{t}(t)=\frac{c_{0} k_{a b}}{k_{a b}-k_{t}}\left[e^{-k_{t} t}-e^{-k_{a b} t}\right], \\
& c_{v b}(t)=c_{0} k_{t} k_{a b}\left[\frac{e^{-k_{t} t}}{\left(k_{a b}-k_{t}\right)\left(k_{c}-k_{t}\right)}-\frac{e^{-k_{a b} t}}{\left(k_{a b}-k_{t}\right)\left(k_{c}-k_{a b}\right)}-\frac{e^{-k_{c} t}}{\left(k_{c}-k_{t}\right)\left(k_{c}-k_{a b}\right)}\right] .
\end{aligned}
$$

\section{Numerical simulation}

The applicability of our method is then tested on mathematical models established in Sect. 4 to obtain the approximate values. For the interval of $t$ as given in Sect. 4, the numerical results that present the maximum error MAXE and the execution time in seconds TIME for all models are given in Tables 4-10 with

$$
\operatorname{MAXE}=\underbrace{\max }_{1 \leq t \leq T}(\underbrace{\max }_{1 \leq i \leq N}\left|\left(y_{i}\right)_{t}-\left(y\left(x_{i}\right)\right)_{t}\right|)
$$


Table 4 Numerical results for concentration of drug in Model A

\begin{tabular}{llll}
\hline $\mathrm{h}$ & Method & MAXE & TIME \\
\hline $10^{-2}$ & $\rho$-DIBBDF & $3.09796 \times 10^{-4}$ & $1.48973 \times 10^{-5}$ \\
& NDIBBDF & $3.56173 \times 10^{-4}$ & $3.97651 \times 10^{-5}$ \\
& BBDF- $\alpha$ & $3.39287 \times 10^{-4}$ & $7.00533 \times 10^{-5}$ \\
& ode15s & $7.75030 \times 10^{-3}$ & $3.40630 \times 10^{-2}$ \\
& ode23s & $4.16650 \times 10^{-3}$ & $5.00000 \times 10^{-2}$ \\
& $\rho$-DIBBDF & $3.26669 \times 10^{-8}$ & $4.80924 \times 10^{-4}$ \\
$10^{-4}$ & NDIBBDF & $3.81740 \times 10^{-8}$ & $5.65695 \times 10^{-3}$ \\
& BBDF- $\alpha$ & $3.61969 \times 10^{-8}$ & $8.25088 \times 10^{-3}$ \\
& ode15s & $1.53220 \times 10^{-4}$ & $6.09380 \times 10^{-2}$ \\
& ode23s & $2.40510 \times 10^{-4}$ & $9.81250 \times 10^{-2}$ \\
& $\rho$-DIBBDF & $5.29902 \times 10^{-11}$ & $2.34869 \times 10^{-2}$ \\
$10^{-6}$ & NDIBBDF & $3.24996 \times 10^{-10}$ & $4.97863 \times 10^{-1}$ \\
& BBDF- $\alpha$ & $2.56585 \times 10^{-10}$ & $9.54964 \times 10^{-1}$ \\
& ode15s & $2.44190 \times 10^{-6}$ & $9.37500 \times 10^{-1}$ \\
& ode23s & $1.16910 \times 10^{-5}$ & $5.46880 \times 10^{-1}$ \\
\hline
\end{tabular}

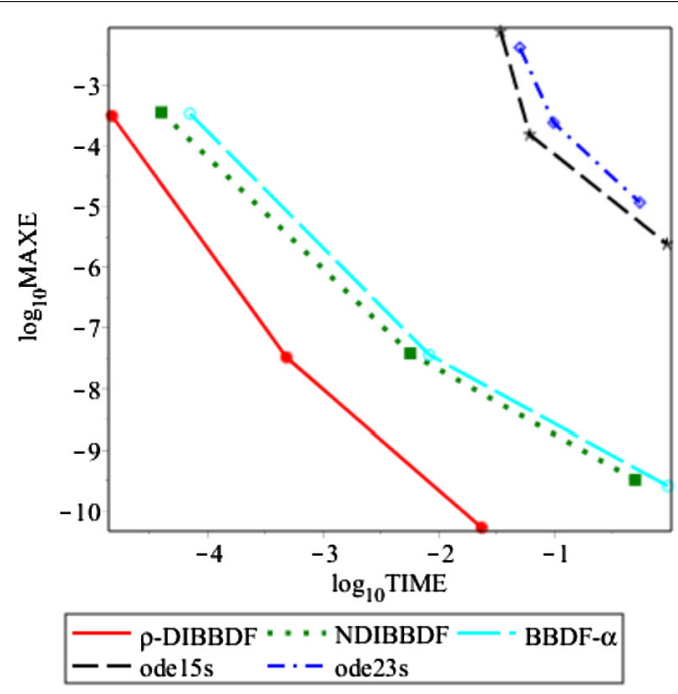

Figure 6 Graph for $\log _{10}$ MAXE against $\log _{10}$ TIME of Model A

Table 5 Numerical results for concentration of drug in Model B-Compound (i)

\begin{tabular}{llll}
\hline $\mathrm{h}$ & Method & MAXE & TIME \\
\hline $10^{-2}$ & $\rho$-DIBBDF & $1.81939 \times 10^{-3}$ & $4.34094 \times 10^{-5}$ \\
& NDIBBDF & $2.05922 \times 10^{-3}$ & $2.06814 \times 10^{-4}$ \\
& BBDF- $\alpha$ & $1.98698 \times 10^{-3}$ & $9.26115 \times 10^{-4}$ \\
& ode15s & $6.71190 \times 10^{-3}$ & $1.40630 \times 10^{-2}$ \\
& ode23s & $3.73690 \times 10^{-3}$ & $4.68750 \times 10^{-2}$ \\
& $\rho$-DIBBDF & $2.04691 \times 10^{-7}$ & $7.38587 \times 10^{-3}$ \\
$10^{-4}$ & NDIBBDF & $2.39095 \times 10^{-7}$ & $1.27894 \times 10^{-2}$ \\
& BBDF- $\alpha$ & $2.26730 \times 10^{-7}$ & $4.04615 \times 10^{-2}$ \\
& ode15s & $1.31340 \times 10^{-4}$ & $5.46880 \times 10^{-2}$ \\
& ode23s & $2.10060 \times 10^{-4}$ & $8.25000 \times 10^{-2}$ \\
& $\rho$-DIBBDF & $2.05082 \times 10^{-11}$ & $2.49705 \times 10^{-1}$ \\
$10^{-6}$ & NDIBBDF & $5.25957 \times 10^{-11}$ & $1.58709 \times 10^{0}$ \\
& BBDF- $\alpha$ & $4.82780 \times 10^{-11}$ & $6.19037 \times 10^{0}$ \\
& ode15s & $1.84520 \times 10^{-6}$ & $8.25000 \times 10^{-1}$ \\
& ode23s & $1.02030 \times 10^{-5}$ & $9.37500 \times 10^{-1}$ \\
\hline
\end{tabular}




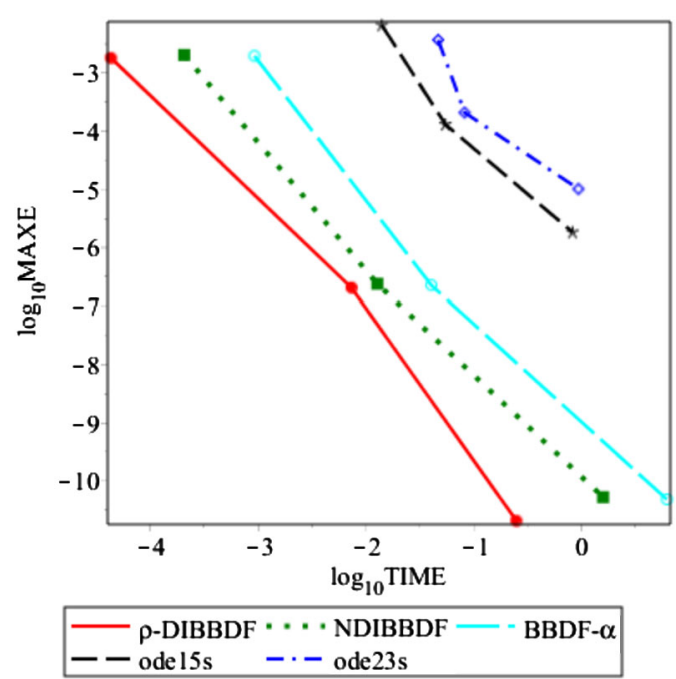

Figure 7 Graph for $\log _{10}$ MAXE against $\log _{10}$ TIME of Model $B(i)$

Table 6 Numerical results for concentration of drug in Model B-Compound (ii)

\begin{tabular}{llll}
\hline $\mathrm{h}$ & Method & MAXE & TIME \\
\hline $10^{-2}$ & $\rho$-DIBBDF & $9.00892 \times 10^{-5}$ & $3.14674 \times 10^{-5}$ \\
& NDIBBDF & $1.04368 \times 10^{-4}$ & $3.13904 \times 10^{-4}$ \\
& BBDF- $\alpha$ & $9.91598 \times 10^{-5}$ & $8.81046 \times 10^{-4}$ \\
& ode15s & $5.32520 \times 10^{-3}$ & $1.09380 \times 10^{-2}$ \\
& ode23s & $3.07010 \times 10^{-3}$ & $1.12500 \times 10^{-2}$ \\
& $\rho$-DIBBDF & $9.30291 \times 10^{-9}$ & $1.68845 \times 10^{-3}$ \\
$10^{-4}$ & NDIBBDF & $1.08726 \times 10^{-8}$ & $4.29541 \times 10^{-2}$ \\
& BBDF- $\alpha$ & $1.03090 \times 10^{-8}$ & $8.75693 \times 10^{-2}$ \\
& ode15s & $1.02730 \times 10^{-4}$ & $5.12500 \times 10^{-2}$ \\
& ode23s & $1.83930 \times 10^{-4}$ & $5.25000 \times 10^{-2}$ \\
& $\rho$-DIBBDF & $3.23822 \times 10^{-11}$ & $7.09676 \times 10^{-1}$ \\
$10^{-6}$ & NDIBBDF & $1.29477 \times 10^{-10}$ & $5.17943 \times 10^{0}$ \\
& BBDF- $\alpha$ & $1.24770 \times 10^{-10}$ & $9.31750 \times 10^{0}$ \\
& ode15s & $1.21500 \times 10^{-6}$ & $6.25000 \times 10^{-1}$ \\
& ode23s & $9.15440 \times 10^{-6}$ & $7.81250 \times 10^{-1}$ \\
\hline
\end{tabular}
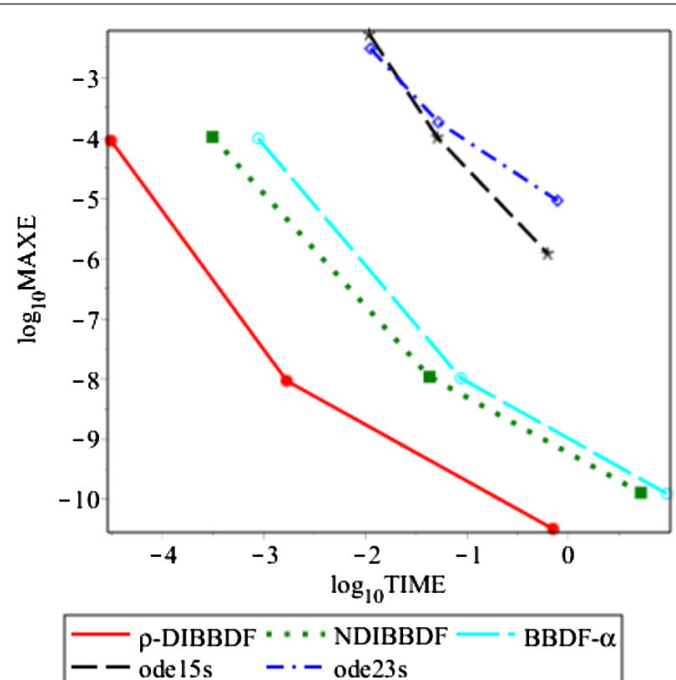

Figure 8 Graph for $\log _{10}$ MAXE against $\log _{10}$ TIME of Model B(ii) 
Table 7 Numerical results for concentration of drug in Model B-Compound (iii)

\begin{tabular}{llll}
\hline $\mathrm{h}$ & Method & MAXE & TIME \\
\hline $10^{-2}$ & $\rho$-DIBBDF & $1.91097 \times 10^{-4}$ & $5.01770 \times 10^{-5}$ \\
& NDIBBDF & $2.20710 \times 10^{-4}$ & $6.30163 \times 10^{-4}$ \\
& BBDF- $\alpha$ & $2.09954 \times 10^{-4}$ & $8.40108 \times 10^{-4}$ \\
& ode15s & $6.80330 \times 10^{-3}$ & $3.12500 \times 10^{-2}$ \\
& ode23s & $3.77840 \times 10^{-3}$ & $3.25000 \times 10^{-2}$ \\
& $\rho$-DIBBDF & $1.99379 \times 10^{-8}$ & $3.45319 \times 10^{-3}$ \\
$10^{-4}$ & NDIBBDF & $2.33007 \times 10^{-8}$ & $4.05777 \times 10^{-2}$ \\
& BBDF- $\alpha$ & $2.20934 \times 10^{-8}$ & $8.22307 \times 10^{-2}$ \\
& ode15s & $1.33260 \times 10^{-4}$ & $1.40630 \times 10^{-1}$ \\
& ode23s & $2.12780 \times 10^{-4}$ & $3.25000 \times 10^{-1}$ \\
& $\rho$-DIBBDF & $4.19052 \times 10^{-11}$ & $4.28401 \times 10^{-1}$ \\
$10^{-6}$ & NDIBBDF & $1.74958 \times 10^{-10}$ & $5.91702 \times 10^{0}$ \\
& BBDF- $\alpha$ & $1.66374 \times 10^{-10}$ & $9.10122 \times 10^{0}$ \\
& ode15s & $1.90470 \times 10^{-6}$ & $9.37500 \times 10^{-1}$ \\
& ode23s & $1.03310 \times 10^{-5}$ & $8.71880 \times 10^{-1}$ \\
\hline
\end{tabular}

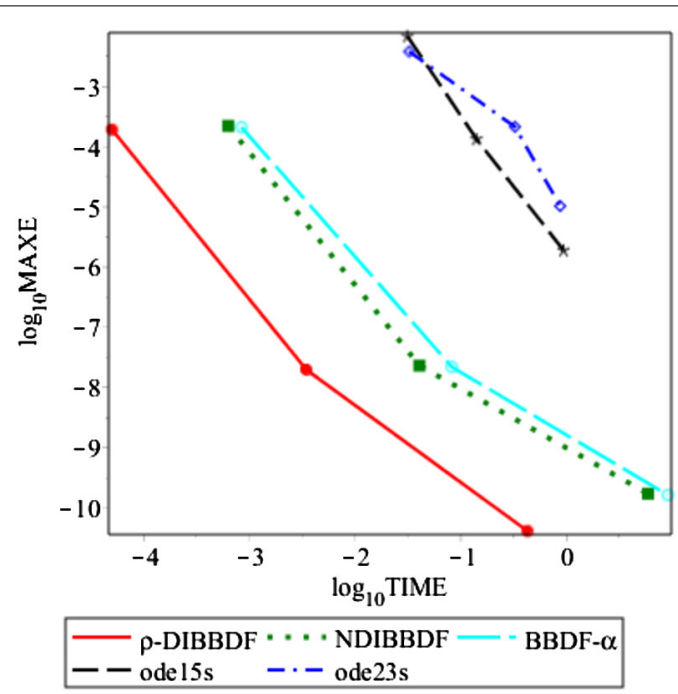

Figure 9 Graph for $\log _{10}$ MAXE against $\log _{10}$ TIME of Model B(iii)

where $t$ is the $t$ th component of the total number of steps; $T, i$ is the $i$ th component of the number of equations; $N$ and $y_{i}$ and $y\left(x_{i}\right)$ are the approximated and exact solutions, respectively. The abbreviations used in Tables $4-10$ are described as follows:

\section{$h$ step length}

$\rho$-DIBBDF $\rho$-diagonally implicit block backward differentiation formula

NDIBBDF new diagonally implicit block backward differentiation formula of order 2 (see [27])

BBDF- $\alpha$ block backward differentiation formula- $\alpha$ with the same back values as our method (see [28])

ode15s quasi-constant step size implementation of the numerical differentiation formula

ode23s modified implicit Rosenbrock methods of order 2

The numerical results of $\rho$-DIBBDF compared to NDIBBDF, BBDF- $\alpha$, Matlab solvers; ode15s and ode23s are displayed in Tables 4-10 for Models A, B, and C. Our results 
Table 8 Numerical results for concentration of drug in Model C(i)

\begin{tabular}{llll}
\hline $\mathrm{h}$ & Method & MAXE & TIME \\
\hline $10^{-2}$ & $\rho$-DIBBDF & $8.69438 \times 10^{-2}$ & $3.51407 \times 10^{-5}$ \\
& NDIBBDF & $1.00483 \times 10^{-1}$ & $6.10869 \times 10^{-5}$ \\
& BBDF- $\alpha$ & $9.55666 \times 10^{-2}$ & $6.06108 \times 10^{-5}$ \\
& ode15s & $8.62070 \times 10^{-1}$ & $1.25000 \times 10^{-2}$ \\
& ode23s & $1.18750 \times 10^{0}$ & $6.25000 \times 10^{-2}$ \\
& $\rho$-DIBBDF & $9.05767 \times 10^{-6}$ & $2.89782 \times 10^{-4}$ \\
$10^{-4}$ & NDIBBDF & $1.05854 \times 10^{-5}$ & $5.31252 \times 10^{-4}$ \\
& BBDF- $\alpha$ & $1.00369 \times 10^{-5}$ & $1.12681 \times 10^{-3}$ \\
& ode15s & $1.78620 \times 10^{-2}$ & $7.12500 \times 10^{-2}$ \\
& ode23s & $5.83780 \times 10^{-2}$ & $2.56250 \times 10^{-1}$ \\
& $\rho$-DIBBDF & $2.24617 \times 10^{-8}$ & $4.18983 \times 10^{-2}$ \\
$10^{-6}$ & NDIBBDF & $1.16015 \times 10^{-7}$ & $1.30586 \times 10^{-1}$ \\
& BBDF- $\alpha$ & $9.98386 \times 10^{-8}$ & $2.20010 \times 10^{-1}$ \\
& ode15s & $2.51080 \times 10^{-4}$ & $2.18750 \times 10^{-1}$ \\
& ode23s & $2.71070 \times 10^{-3}$ & $8.65630 \times 10^{-1}$ \\
\hline
\end{tabular}

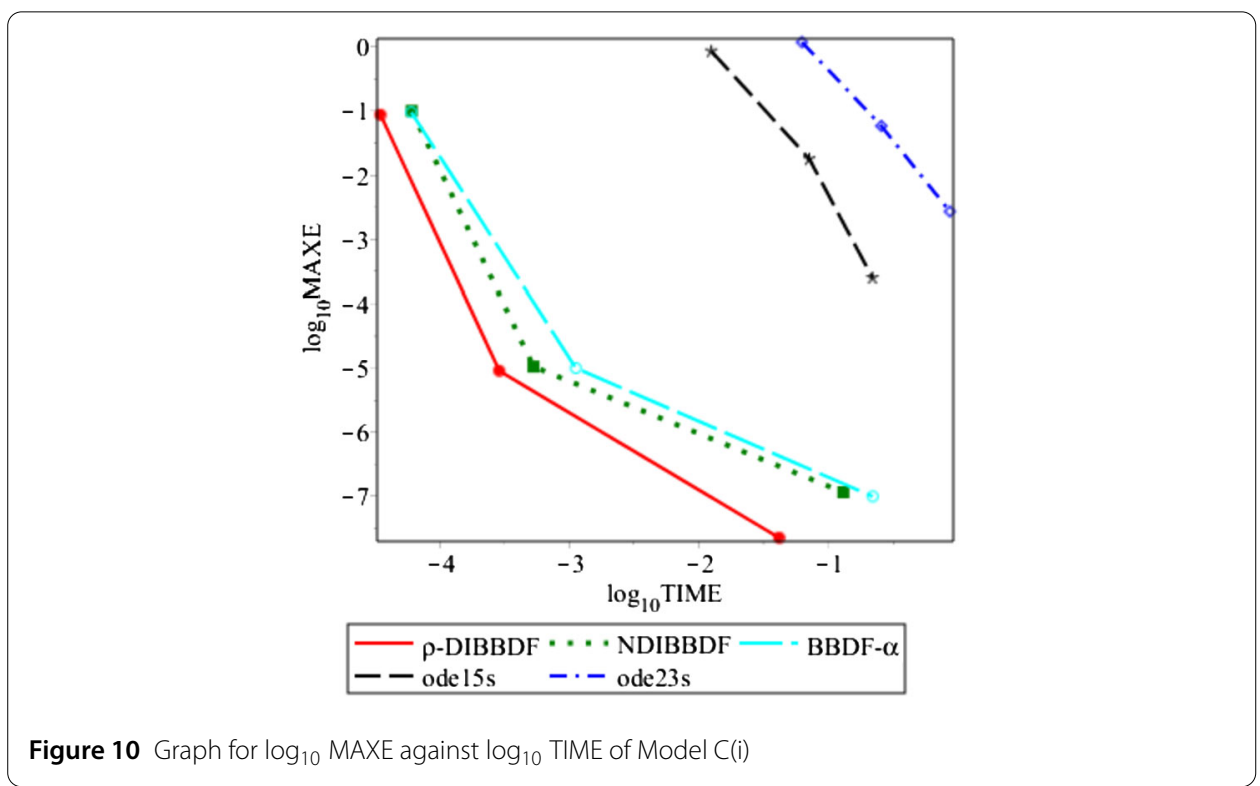

demonstrate that for step lengths smaller than $10^{-2}$ in Tables 8 , 9, and 10, our method gives better performance in terms of efficiency and accuracy compared to other methods. However, for Model C, the numerical results produced slightly larger MAXE when $h=10^{-2}$. As discussed in Sect. 3 , this is because absolute stability will only be achieved if $h \lambda<0.003096032803$. Since the largest $\lambda$ for Model C(i) is $|\lambda|=0.9776$, then $h$ is required to be less than $3.1669 \times 10^{-3}$. A similar constraint also happened in Models $C($ ii) and C(iii), where $h$ is restricted to $2.0935 \times 10^{-3}$ and $3.1669 \times 10^{-3}$, respectively. It is important to highlight that it is the demand for accuracy and not for linear stability that restricts the step length.

To observe the performance of our method, graphs of $\log _{10}$ MAXE against $\log _{10}$ TIME for Models A, B, and C are depicted individually in Figs. 6-12. The performance graphs indicate that the proposed scheme has a uniform pattern of error scaled, which is a significant improvement compared with existing numerical methods. In terms of the execution 
Table 9 Numerical results for concentration of drug in Model C(ii)

\begin{tabular}{llll}
\hline $\mathrm{h}$ & Method & MAXE & TIME \\
\hline $10^{-2}$ & $\rho$-DIBBDF & $1.28576 \times 10^{-1}$ & $2.62771 \times 10^{-5}$ \\
& NDIBBDF & $1.47664 \times 10^{-1}$ & $5.03962 \times 10^{-5}$ \\
& BBDF- $\alpha$ & $1.40710 \times 10^{-1}$ & $3.68505 \times 10^{-4}$ \\
& ode15s & $8.07060 \times 10^{-1}$ & $2.37500 \times 10^{-2}$ \\
& ode23s & $1.18000 \times 10^{0}$ & $3.46880 \times 10^{-2}$ \\
& $\rho$-DIBBDF & $1.35922 \times 10^{-5}$ & $2.71888 \times 10^{-4}$ \\
$10^{-4}$ & NDIBBDF & $1.58834 \times 10^{-5}$ & $7.83104 \times 10^{-4}$ \\
& BBDF- $\alpha$ & $1.50609 \times 10^{-5}$ & $1.09721 \times 10^{-3}$ \\
& ode15s & $1.91560 \times 10^{-2}$ & $9.81250 \times 10^{-2}$ \\
& ode23s & $6.17390 \times 10^{-2}$ & $8.81250 \times 10^{-2}$ \\
& $\rho$-DIBBDF & $2.21650 \times 10^{-8}$ & $1.89351 \times 10^{-2}$ \\
$10^{-6}$ & NDIBBDF & $1.70972 \times 10^{-7}$ & $3.69889 \times 10^{-2}$ \\
& BBDF- $\alpha$ & $1.38956 \times 10^{-7}$ & $4.49960 \times 10^{-2}$ \\
& ode15s & $2.94160 \times 10^{-4}$ & $7.81250 \times 10^{-1}$ \\
& ode23s & $2.91030 \times 10^{-3}$ & $2.87500 \times 10^{-1}$ \\
\hline
\end{tabular}

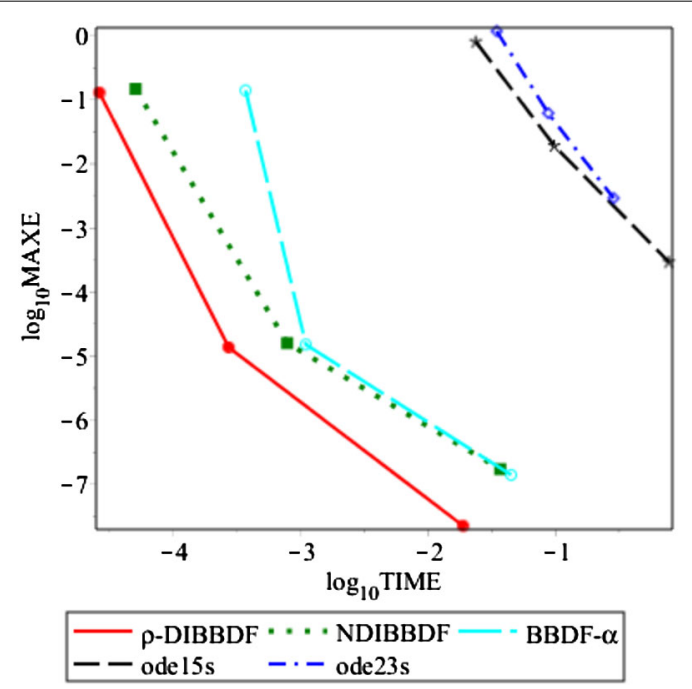

Figure 11 Graph for $\log _{10}$ MAXE against $\log _{10}$ TIME of Model C(ii)

time, Figs. 6-12 display that the $\rho$-DIBBDF method is capable of performing faster than the comparing methods for all models.

\section{Conclusion}

This study has presented a two-point block method based on BDF in a diagonally implicit structure that holds an $A$-stable property. Stability analysis showed that the proposed method is zero stable, absolutely stable, and convergent. The relevant proof that the method satisfies the condition of stiff stability was also provided. The results provide a basis for the effect of the increase in error due to the step length that operates outside the stability region. In conclusion, the suggested method appeared to perform better than the existing methods of NDIBBDF (formulated in a diagonally implicit manner), BBDF- $\alpha$ (implemented in a fully implicit manner), and the Matlab solvers. Hence, $\rho$-DIBBDF is significant to serve as an efficient numerical method and as an alternative solver for the 
Table 10 Numerical results for concentration of drug in Model C(iii)

\begin{tabular}{llll}
\hline $\mathrm{h}$ & Method & MAXE & TIME \\
\hline $10^{-2}$ & $\rho$-DIBBDF & $9.46454 \times 10^{-2}$ & $1.04955 \times 10^{-5}$ \\
& NDIBBDF & $1.09318 \times 10^{-1}$ & $8.06114 \times 10^{-5}$ \\
& BBDF- $\alpha$ & $1.03989 \times 10^{-1}$ & $7.78064 \times 10^{-5}$ \\
& ode15s & $2.82090 \times 10^{-1}$ & $2.25000 \times 10^{-2}$ \\
& ode23s & $8.81200 \times 10^{-1}$ & $1.12500 \times 10^{-2}$ \\
& $\rho$-DIBBDF & $9.87337 \times 10^{-6}$ & $4.15497 \times 10^{-4}$ \\
$10^{-4}$ & NDIBBDF & $1.15386 \times 10^{-5}$ & $1.92512 \times 10^{-3}$ \\
& BBDF- $\alpha$ & $1.09407 \times 10^{-5}$ & $9.40735 \times 10^{-4}$ \\
& Ode15s & $8.64250 \times 10^{-3}$ & $9.37500 \times 10^{-2}$ \\
& ode23s & $4.19090 \times 10^{-2}$ & $1.09380 \times 10^{-1}$ \\
& $\rho$-DIBBDF & $2.01807 \times 10^{-8}$ & $8.80850 \times 10^{-2}$ \\
$10^{-6}$ & NDIBBDF & $1.36344 \times 10^{-7}$ & $1.24745 \times 10^{0}$ \\
& BBDF- $\alpha$ & $1.09823 \times 10^{-7}$ & $1.07228 \times 10^{-1}$ \\
& ode15s & $1.59290 \times 10^{-4}$ & $3.12500 \times 10^{-1}$ \\
& ode23s & $1.93740 \times 10^{-3}$ & $5.50000 \times 10^{-1}$ \\
\hline
\end{tabular}

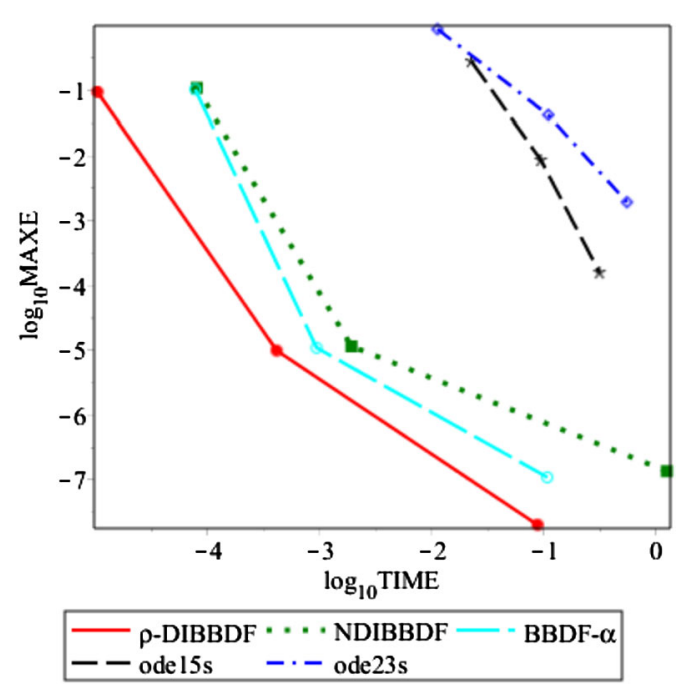

Figure 12 Graph for $\log _{10}$ MAXE against $\log _{10}$ TIME of Model C(iii)

pharmacokinetics model of ODEs. For future research, $\rho$-DIBBDF may also be applied to other models arising in the pharmacokinetics field.

\section{Acknowledgements}

The authors would like to thank the editor and reviewers for the constructive comments and useful suggestions, which improved the manuscript.

\section{Funding}

This research was funded by Universiti Putra Malaysia under Grant Putra, project number GP/2018/9650600.

Availability of data and materials

Not applicable.

Competing interests

The authors declare that they have no competing interests.

Authors' contributions

All authors contributed equally to the manuscript and read and approved the final manuscript. 


\section{Publisher's Note}

Springer Nature remains neutral with regard to jurisdictional claims in published maps and institutional affiliations.

\section{Received: 1 March 2020 Accepted: 21 July 2020 Published online: 05 August 2020}

\section{References}

1. Gear, C.W.: Algorithm 407: DIFSUB for solution of ordinary differential equations. Commun. ACM 14, 185-190 (1971)

2. Enright, W.H.: Second derivative multistep methods for stiff ordinary differential equations. SIAM J. Numer. Anal. 11, 321-331 (1974)

3. Lambert, J.D.: Computational Methods in Ordinary Differential Equations. Wiley, London (1973)

4. Lapidus, L., Seinfeld, J.H.: Numerical Solution of Ordinary Differential Equations. Academic Press, New York (1971)

5. Lambert, J.D.: Numerical Methods for Ordinary Differential Systems: The Initial Value Problem. Wiley, New York (1991)

6. Kennedy, C.A., Carpenter, M.H.: Diagonally implicit Runge-Kutta methods for stiff ODEs. Appl. Numer. Math. 146, 221-244 (2019)

7. Skvortsov, L.M.: Diagonally implicit Runge-Kutta methods for stiff problems. Comput. Math. Math. Phys. 46 , 2110-2123 (2006)

8. Alexander, R.: Diagonally implicit Runge-Kutta methods for solving stiff O.D.E'S. SIAM J. Numer. Anal. 14, 1006-1021 (1977)

9. Norsett, S.P.: Semi-Explicit Runge-Kutta Methods. Report Mathematics and Computation, No. 6/74. Dept. of Mathematics, Universiti of Trondheim, Norway (1974)

10. Piao, X.F., Choi, H., Kim, S.D.: A fast singly diagonally implicit Runge-Kutta method for solving 1D unsteady convection-diffusion equations. Numer. Methods Partial Differ. Equ. 30, 788-812 (2013)

11. Kim, P., Piao, X., Jung, W., Bu, S.: A new approach to estimating a numerical solution in the error embedded correction framework. Adv. Differ. Equ. 2018, 168 (2018)

12. Homayun, B., Lin, X., Choi, H.J.: Challenges and recent progress in oral drug delivery systems for Biopharmaceuticals. Pharm. 11, 129 (2019)

13. Ensign, L.M., Cone, R., Hanes, J.: Oral drug delivery with polymeric nanoparticles: the gastrointestinal mucus barriers. Adv. Drug Deliv. Rev. 64, 557-570 (2012)

14. Sastry, S.V., Nyshadham, J.R., Fix, J.A.: Recent technological advances in oral drug delivery-a review. Pharm. Sci. Technol. Today 3, 138-145 (2000)

15. Savjani, K.T., Gajjar, A.K., Savjani, J.K.: Drug solubility: importance and enhancement techniques. ISRN Pharm. 2012 $195727(2012)$

16. Dey, P., Maiti, S.: Orodispersible tablets: a new trend in drug delivery. J. Nat. Sci. Biol. Med. 1, 2-5 (2010)

17. Bischoff, K.B., Dedrick, R.L.: Generalized solution to linear, two-compartment, open model for drug distribution. J. Theor. Biol. 29, 63-83 (1970)

18. Jacobs, J.R.: Analytical solution to the three-compartment pharmacokinetic model. IEEE Trans. Biomed. Eng. 35, 763-765 (1988)

19. Spitznagel, E.: Two-Compartment Pharmacokinetics Models. C-ODE-E. Harvey Mudd College, Fall (1992)

20. Shonkwiler, R.W., Herod, J.: Mathematical Biology: An Introduction with Maple and Matlab. Springer, Berlin (2009)

21. Shityakov, S., Förster, C.: Pharmacokinetic delivery and metabolizing rate of Nicardipine incorporated in Hydrophilic and Hydrophobic Cyclodextrins using two-compartment mathematical model. Sci. World J. 2013, 131358 (2013)

22. Chomcheon, S., Lenbury, Y., Sarika, W.: Stability, Hopf bifurcation and effects of impulsive antibiotic treatments in a model of drug resistance with conversion delay. Adv. Differ. Equ. 2019, 274 (2019)

23. Khanday, M.A., Rafiq, A., Nazir, K.: Mathematical models for drug diffusion through the compartments of blood and tissue medium. Alexandria J. Med. 11, 245-249 (2017)

24. Ibrahim, Z.B., Othman, K.I., Suleiman, M.B.: Implicit $r$-point block backward differentiation formula for solving first-order stiff ODEs. Appl. Math. Comput. 186, 558-565 (2007)

25. Suleiman, M.B., Musa, H., Ismail, F., Senu, N., Ibrahim, Z.B.: A new superclass of block backward differentiation formula for stiff ordinary differential equations. Asian-Eur. J. Math. 7, 1-17 (2014)

26. Zawawi, I.S.M., Ibrahim, Z.B., Othman, K.I.: Derivation of diagonally implicit block backward differentiation formulas for solving stiff initial value problems. Math. Probl. Eng. 2015, Article ID 179231 (2015)

27. Babangida, B., Musa, H., Ibrahim, L.K.: A new numerical method for solving stiff initial value problems. Fluid Mech. Open Access 3, 136 (2016)

28. Zawawi, I.S.M., Ibrahim, Z.B.: Derivation of BBDF- $\alpha$ for solving ordinary differential equations. AIP Conf. Proc. 1750, $020018(2016)$

29. Dahlquist, G.: A special stability problem for linear multistep methods. BIT Numer. Math. 3, 27-43 (1963)

30. Butcher, J.C.: Forty-five years of A-stability. J. Numer. Anal. Ind. Appl. Math. 4, 1-9 (2009)

31. Liniger, W.: A criterion for A-stability of linear multistep integration formulae. Computing 3, 280-285 (1968)

32. Vijitha-Kumara, K.H.Y.: Variable stepsize variable order multistep methods for stiff ordinary differential equations. PhD thesis, lowa State University, Ames, IA, USA (1985)

33. Rockswold, G.K.: Stable variable step stiff methods for ordinary differential equations. PhD thesis, lowa State University, Ames, IA, USA (1983)

34. Hall, G., Watt, J.M.: Modern Numerical Methods for Ordinary Differential Equations. Clarendon, Oxford (1976)

35. Ijam, H.M., Ibrahim, Z.B.: Diagonally implicit block backward differentiation formula with optimal stability properties for stiff ordinary differential equations. Symmetry 11, 1342 (2019)

36. Henrici, P.: Discrete Variable Methods in Ordinary Differential Equations. Wiley, New York (1962)

37. Aksah, S.J., Ibrahim, Z.B., Zawawi, I.S.M.: Stability analysis of singly diagonally implicit block backward differentiation formulas for stiff ordinary differential equations. Mathematics 7, 211 (2019)

38. Borrelli, R.L., Coleman, C.S.: Differential Equations: A Modeling Perspective. Wiley, New York (2004) 